\title{
Strategie ed efficacia della medicina narrativa nei pazienti nefrologici: una revisione della letteratura. Parte 1
}

\author{
Mariangela Mettifogo ${ }^{1}$, Francesco Burrai ${ }^{2,3}$, Valentina Dorigo ${ }^{4}$, Carla Zampieri ${ }^{1}$ \\ ${ }^{1}$ Dipartimento di Nefrologia, Dialisi e Trapianto, Ospedale San Bortolo, Vicenza \\ ${ }^{2}$ Servizio Professioni Sanitarie, ATS Sardegna-ASSL di Olbia, Olbia \\ ${ }^{3}$ Direttore Scientifico dell'Italian EDTNA/ERCA Research Center, Bologna \\ ${ }^{4}$ U.O.S Dialisi, Ospedale di Dolo, Venezia
}

\begin{abstract}
Strategies and effectiveness of narrative medicine in renal patients: a literature review. Part 1
Introduction: This paper on narrative-based medicine (NBM) is divided into two articles. The concept of NBM has been developed over the last decades to bring the biomedical aspects of illness and treatment closer to the psychosocial aspects and life experiences related to illness.

Purpose: To identify and summarize the evidence and concepts of NBM in nephrology.

Methods: Review of studies based on a literature search in 6 databases.

Results: Thirty-five papers were included in the review, none of which were quantitative.

Discussion: The narrative approach applied to a nephrology context seeks to shed light on the life experiences related to kidney disease by collecting, through structured and semistructured interviews, stories from patients, caregivers and health care providers. Some of the main topics emerging in the first article are the lack of a "disease identity" that allows patients to identify with the specific status of kidney disease; uncertainty as a dominant feature of living with an illness characterized by continuous progression and regression; the "unspeakable", referring to all those aspects for which there is no room for expression in the care relationship, such as living with the risk of dying, experiences that are difficult to put into words, and everything related to mystery, faith and the inexplicable.

Conclusions: This review shows the importance of exploring the disturbing aspects of life for patients with chronic renal failure. Knowledge of these aspects can help health care providers improve the quality and effectiveness of the therapeutic relationship and ensure that the patient will not feel isolated but at the center of the care process. Narrative medicine, as will be highlighted in the second article, is also aimed at health care providers in order to investigate their experiences, which are often characterized by situations of suffering and discomfort. The narrative approach in nephrology could be supported by further research into the therapeutic abilities of narration in the care relationship.

Keywords: Narration, Narrative medicine, Narrative nursing
\end{abstract}

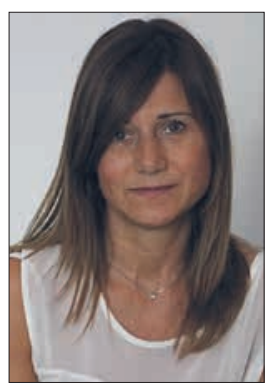

Mariangela Mettifogo

\section{Introduzione}

La Narrative Based Medicine (NBM) si basa su una complessa concezione di malattia, fondata sul concetto di integrazione mente-corpo su cui intervengono influenze contestuali, socialmente, culturalmente e storicamente determinate, con le conseguenti caratteristiche di relatività e di incertezza che ne caratte-

Accepted: August 3, 2017

Published online: November 14, 2017

Indirizzo per la corrispondenza:

Mariangela Mettifogo

Dipartimento di Nefrologia, Dialisi e Trapianto renale

Ospedlae San Bortolo - ULSS 8 Berica

Viale Rodolfi, 37 - 36100 Vicenza

mariangela.mettifogo@gmail.com rizzano l'orientamento. Nei primi studi ermeneutico-fenomenologici, la medicina è stata considerata un sistema culturale i cui significati simbolici modellano il vissuto del malato (1), e la narrazione la modalità con cui la persona attribuisce significato agli eventi e si orienta rispetto alle azioni e alle scelte da intraprendere. Secondo l'approccio ermeneutico la condivisione della pluralità dei significati che emergono dall'incontro tra sanitario e paziente può contribuire a una co-costruzione di senso dell'esperienza vissuta. La narrazione da parte del malato della propria storia e l'ascolto di chi si prende cura di lui, gli permettono di esprimere il proprio disagio e le proprie emozioni, di instaurare una relazione terapeutica efficace, che lo aiuti a prendere le decisioni in modo più consapevole e a esserne più partecipe. Gli studi scientifici presenti in letteratura, svolti nell'ambito della medicina narrativa, sono per lo più di tipo qualitativo e si basano sulla prospettiva costruttivista che parte dal presupposto che persone, gruppi e culture creano e sostengono la loro comprensione e rappresentazione della realtà attraverso la costruzione condivisa di storie (2). Il Nursing Narrativo nasce con gli studi di Paterson e Zderad 
(3) che, riprendendo alcuni concetti fenomenologici di Husserl, considerano il vissuto esperienziale come ciò che scaturisce dalla condivisione, da parte del paziente e dell' infermiere, di una stessa dimensione spaziale e temporale. Fare Infermieristica Narrativa significa valorizzare la narrazione di questo vissuto, attraverso la scrittura, la parola, la poesia, il disegno, la fotografia.

\section{Metodi di ricerca}

L'obiettivo di questo studio è stato quello di individuare e sintetizzare le evidenze e i concetti della NBM in campo nefrologico. È stata eseguita una revisione della letteratura consultando le seguenti sei banche dati informatizzate: Cochrane Library, PubMed, MEDLINE, Embase, Cinahl, PsycINFO. Per la ricerca degli studi sono state identificate alcune specifiche parole chiave, quali narrative medicine, narrative nursing, narrative, nursing, kidney renal care, dialysis, nefrology. I criteri di inclusione sono stati i seguenti: articoli full text, in inglese, di tipo quantitativo, qualitativo e mixed method, il cui topic era la NBM nel campo della nefrologia. Gli articoli trovati, sia abstract sia full text, sono stati tutti tradotti e analizzati.

\section{Risultati}

La revisione effettuata ha permesso di recuperare 35 studi sull'argomento oggetto di ricerca, dai quali sono emersi importanti temi relativi alla malattia renale che descriveremo in questo e nel successivo articolo. In questo articolo verranno affrontati alcuni particolari vissuti del paziente nefrologico spesso caratterizzati dalla comparsa di sintomi indefiniti e vaghi, da un'evoluzione non lineare e continua, bensì interrotta da frequenti regressioni dovute alla comorbilità spesso presente nel decorso di questa malattia. Emergeranno vissuti di incertezza ed esperienze difficili da verbalizzare.

Nel prossimo articolo vedremo come la narrazione, grazie anche a modalità di espressione alternative e complementari al linguaggio verbale, possa essere efficace nell'elaborazione della propria sofferenza e nell'affrontare temi etici importanti; come alcuni concetti nati in ambito antropologico (liminalità) e filosofico (campo e habitus) applicati in ambito medico possano fornire una chiave di lettura per alcuni particolari processi che si manifestano nel contesto della malattia e della cura.

\section{Approcci teorici sulla malattia renale}

Il costrutto teorico che lega insieme i vari temi emersi da questa revisione, sembra essere la considerazione della malattia non solo ed esclusivamente come un semplice danno d'organo, ma come un processo dinamico in cui entrano in causa fattori socioculturali che influiscono sul modo in cui la persona malata, la sua famiglia e la società percepiscono, vivono e rispondono ai sintomi e alla disabilità (4). Comprendere gli esseri umani in maniera olistica vuol dire quindi considerare il paziente come una persona con una propria storia. La Medicina Narrativa permette di far emergere la storia individuale del paziente, ma anche di evidenziare alcuni contenuti che accomunano e caratterizzano un certo tipo di esperienza. A differenza di altre malattie croniche, come ad esempio il cancro, Vélez et al (4) hanno evidenziato come nell' insufficienza renale cronica ci sia una forte mancanza di un'identità di malattia, ossia quell'insieme di rappresentazioni in base alle quali viene attribuita l'appartenenza a un particolare status. Questo fenomeno sembra essere spiegato da alcuni aspetti tipici di questa patologia, di cui gli unici referenti esplicativi sono la dialisi e il conseguente cambiamento dello stile di vita: tali aspetti si riferiscono all'esordio della malattia, asintomatico o con sintomi poco specifici, alla sua scarsa "popolarità", al fatto che il rene non sia un organo centrale ma uno di una coppia di organi. In uno studio della revisione (5), analizzando le esperienze dei pazienti con oltre vent'anni di dialisi e delle loro famiglie, sono state descritte tre fasi distinte nella vita di queste persone: fase iniziale o di crisi (fino a un anno); fase di vita cronica (fino al quindicesimo anno); fase terminale (quando le condizioni di salute si deteriorano). In un altro studio della revisione (4) emergono altre specifiche fasi della malattia: Fase pre-diagnosi - Fase di diagnosi - Fase di incorporazione - Trapianto/Morte. Emerge in questi articoli che la malattia renale, pur essendo descritta in una sequenza di fasi e stadi, non ha però un decorso lineare ed è spesso caratterizzata da avanzamenti e regressioni, alterazioni e particolarità diverse e personali. Le categorie ontologiche che vengono a crearsi, bloccano i pazienti in particolari modi di essere (per esempio, paziente renale, paziente in dialisi, paziente trapiantato), proponendosi come unità discrete di esperienza che ci danno delle spiegazioni normative sulla malattia. Questo maschera l'esperienza più completa ed esaustiva del malato a favore di una visione riduttiva del corpo come entità cyborg, un insieme assemblato di pezzi di ricambio. La concezione medica di "trapianto", per esempio, come successo terapeutico e tappa finale della malattia, come dono della vita, tiene il malato intrappolato e bloccato e viene quasi completamente sovvertita. Le narrazioni dei pazienti, infatti, smantellano questo tipo di costruzione teorica in quanto essi percepiscono l'assenza dolorosa di una "fine" del loro percorso di malattia, costretti a sopportare una pesante terapia immunosoppressiva nel costante e silenzioso timore di un rigetto. II concetto di dono richiama quello di reciprocità (6) e nei pazienti questa visione suscita la sensazione di essere un ricevitore nobile, il senso di colpa per qualcuno che è morto per il loro bene e inoltre, nei caregivers e nei familiari, la difficoltà a comprendere le problematiche che derivano da questo vissuto. Nei pazienti che hanno una lunga esperienza di dialisi, emergono una serie di "self affirmations" (7) che accompagnano la ristrutturazione di sé e dell'esperienza di malattia. Queste autoaffermazioni, riportate di seguito si riferiscono alla volontà di vivere, al riconoscimento della propria identità, del proprio valore e dell'autocontrollo:

$$
\text { - self-preservation: "I want to live" (Voglio vivere) }
$$$$
\text { - self-identity: "I am still me" (Sono comunque io) }
$$ 
- self-worth: "I am still valuable" (Valgo ancora qualcosa) - self-efficacy: "I am in control” (È tutto sotto controllo).

Questi pazienti convivono con l'incertezza e il rischio di morte (Risk of death/uncertain future), devono adattarsi alle limitazioni imposte dalla malattia (Life constraints) relative all'intrusività della dialisi nella loro vita (Dialysis itself), e vivono frequenti battute d'arresto dovute all'elevata morbilità (Repeated setbacks). L'incertezza viene descritta come componente integrante dell'esperienza di malattia (8); compare con ricorrenti flussi e riflussi nel corso della vita del malato, rendendo l'equilibrio tra il concentrarsi sul vivere e la costante consapevolezza della propria mortalità, molto difficile e delicato. In un altro studio della revisione di Polaschek (5), attraverso dieci interviste semi-strutturate a pazienti, persone di supporto e professionisti della salute, sono state evidenziate delle disparità di visione tra le diverse figure, ambiguità tra la promozione dell'autogestione e la richiesta di adesione terapeutica, tra l'obiettivo dei professionisti di migliorare lo stato di salute dei pazienti e l'obiettivo dei pazienti di vivere una vita normale. Dal 2002, la malattia renale è stata ripensata come una condizione cronica da gestire medicalmente riducendo al minimo i fattori di rischio che possono portare a episodi acuti come la dialisi o il trapianto (National Kidney Foundation [NKF], 2002). Questo ha incentivato un approccio precoce e preventivo alla malattia, che ha riposto l'attenzione sullo stile di vita, l'alimentazione e quindi sul concetto di "vivere bene". Il divario esistente tra la visione del paziente e quella dei sanitari complica la nozione di cosa voglia dire "vivere bene" con la MRC. Una relazione basata sulla fiducia e l'ascolto sembra ridurre queste lacune.

La comunicazione regolare e positiva tra pazienti e operatori è un aspetto importante della cura e dell'empowerment del paziente (9). La pratica narrativa sembra influire sul modo in cui i pazienti vivono e affrontano emotivamente la malattia. Si evidenzia l'importanza che gli operatori si concentrino sui progressi del paziente, annullando il giudizio morale nei confronti dei fallimenti relativi alla gestione della malattia, come la crescita ponderale, l'assunzione dei farmaci e l'alimentazione. Saper dosare lode e sostegno sembra essere un fattore indispensabile per non rischiare di scivolare in un atteggiamento moralistico circa la capacità del paziente di "vivere bene", che produce in lui sensi di colpa, risentimento, e aspettativa di un giudizio e una critica da parte dell'esterno. Questa non è esclusiva responsabilità del professionista ma di un sistema culturale nel quale salute e malattia sono purtroppo ancora collegate ai concetti di bene e male.

\section{Il corpo come mediatore}

Superando le categorie teoriche precostituite di malattia renale cronica, Kierans (10) esplora il vissuto della malattia come un significato mediato dal corpo e dai sensi, influenzato dalla cultura e dai rapporti sociali. Nel suo articolo emerge come la malattia renale sia caratterizzata da particolari esperienze sensoriali che al momento della diagnosi si spostano dall'area per- sonale a quella medica, acquisendone definizione e causalità. Gli eventi che vengono raccontati tracciano una traiettoria di malattia a volte coerente e continua, altre, un susseguirsi di situazioni che rimbalzano il paziente avanti e indietro nel decorso della sua vita, rendendo la prevedibilità del suo futuro incerta e complicata dal rischio di morte che la malattia stessa comporta. Come altre condizioni croniche, l'insufficienza renale sposta I'interiorità del corpo all'esterno, rendendo visibili e percepibili sensazioni e funzioni (10). I pazienti con malattie renali hanno un vissuto fenomenologico della loro malattia molto particolare, basti pensare alla percezione continua e costante del thrill della fistola, che si materializza nel flusso di sangue che essi vedono circolare al di fuori del proprio corpo e scomparire nel circuito della macchina, tornando reintegrato e depurato. Non è il corpo come entità materiale e fissa soggetta alle regole empiriche delle scienze biologiche (6), a emergere. Si tratta di un corpo alterato e privato delle funzioni di sostegno vitale e sociale di cui necessita. Le normali funzioni vitali come mangiare, bere, dormire, urinare non sono più momenti abituali e consuetudini nella vita di tutti i giorni. Le narrazioni dei pazienti evidenziano l'importanza e la centralità del corpo, quale spazio su cui si costruisce e ricostruisce il significato e l'esperienza di malattia, plasmata dagli interventi medici e tecnologici nonché dalle concezioni socialmente condivise, come per esempio la considerazione diffusa e consolidata del trapianto come "dono" che abbiamo affrontato nel paragrafo precedente. La malattia non è qualcosa che "avviene" semplicemente, ma si forma attraverso una "processo di creatività umana" e un "powerful self-process". Nell'insufficienza renale, una moltitudine di singoli eventi e istanze fanno sì che l'esperienza di malattia venga trasformata e resa significativa per i pazienti. Interventi ed esami medici, secondo alcuni autori, hanno la capacità di minacciare il sé incarnato, il senso di unicità organica, dando luogo a processi di "disincarnazione" e pratiche di "disumanizzazione" (6). Ciò conduce al traguardo finale del corpo cyborg, che tuttavia, può essere sperimentato come un nuovo modo di estendere i confini delle forme di realizzazione possibili. Donna Haraway evoca una visione del futuro in cui “... un mondo cyborg esprime l'effetto di una realtà sociale in cui le persone non hanno paura della loro parentela con animali e macchine, non hanno paura di identità permanenti o parziali e punti di vista contraddittori" (6). L'elevata medicalizzazione e tecnicizzazione dell'assistenza e della cura, caratteristiche di questo tipo di malattia inducono una riflessione su questo concetto, che seppur in apparenza lontano dalla realtà ne esprime tutta la particolare complessità.

\section{L'indicibile della malattia renale}

Le narrazioni dei pazienti con malattia renale, comuni a quelle di altre patologie che mettono a rischio la vita, come il cancro e l'AIDS, sono caratterizzate da temi definiti "unsayable" ossia "indicibili" (11), in cui rientrano tutti quegli aspetti che non trovano spazio di espressione nella relazione di cura, come la convivenza con il rischio di morire, tutto ciò 
che è legato al mistero, alla fede, tutto ciò che è inspiegabile e/o difficile da verbalizzare. In una cultura di negazione della morte, questo tema viene spesso evitato così da lasciare muti dubbi, paure, indecisioni sul proprio processo di cura e sull'evoluzione della malattia. A fianco a questo tema, sembrano restare inespresse particolari manifestazioni di dolore fisico, esperienze di pre-morte e anche situazioni di rischio e scarsa sicurezza vissute nell'ambiente ospedaliero. Temi legati ad aspetti spirituali, alla fede religiosa e ai propri valori morali ed etici, evocati e suscitati dalle situazioni di sofferenza, sono spesso taciuti e non affrontati.

Nella maggior parte delle ricerche antecedenti al 2000, fatta eccezione per alcuni autori (2), le parole "religione" e "spiritualità" sono state usate in modo intercambiabile. Oggi si è giunti a un generale accordo sul fatto che si tratti di due costrutti molto diversi non solo a livello concettuale, ma anche a livello individuale. Paley (12), sostiene che gli infermieri non devono considerare i bisogni spirituali come universali in quanto, spiritualità e religione differiscono notevolmente in tutto il mondo ed esiste un'ampia variabilità nel sistema di credenze personali che non sempre rientra in specifiche categorie sociali e religiose. II contesto di cura renale e le relazioni che si creano al suo interno, sembrano essere caratterizzati in maniera distintiva da una sorta di silenzio strutturato, concetto espresso da Zola (13) come "illness as lived in structured silence", descritto come una mancata considerazione della prospettiva del paziente da parte del personale sanitario, che rimane quindi inosservata e incompresa. II linguaggio delle parole sembra non essere sempre adatto a esplicitare e articolare queste esperienze, pur essendo tuttavia l'ascolto di tutti gli aspetti dicibili e indicibili del vivere con la malattia, un'importante competenza di tipo infermieristico.

\section{Discussione}

I risultati emersi da questo lavoro di revisione della letteratura hanno permesso di delineare alcuni importanti aspetti della malattia renale e del modo in cui l'approccio narrativo è stato utilizzato in ambito nefrologico. L'intervista narrativa emerge come metodo di ricerca e raccolta delle narrazioni dei soggetti, siano essi pazienti, familiari o personale sanitario, quest'ultimo abbiamo potuto notare come sia stato molto meno indagato e studiato. Ciò che emerge in modo abbastanza evidente è che, se da un lato i contributi teorici e concettuali presenti in letteratura (clinici, sociologici, antropologici, psicologici) permettono di delineare alcuni aspetti generali della malattia renale, dall'altro, il metodo narrativo permette di far emergere il vissuto soggettivo delle persone, che non deve essere dimenticato e dato per scontato, e consente la personalizzazione delle cure e delle scelte terapeutiche. Nelle malattie nefrologiche, la terapia conservativa e sostitutiva della funzionalità renale dovrebbe essere scelta non solo sulla base di specifici parametri di carattere clinico, ma anche in riferimento alle rappresentazioni dei vissuti del paziente, secondo i quali egli elabora, reagisce e affronta la sua situazione patologica e i conseguenti cambiamenti della propria vita. Solo in questo modo è possibile favorire il successo terapeutico, la partecipazione e un'efficace compliance da parte del paziente. Non sono presenti in questo e nel prossimo articolo della nostra revisione risultati che evidenzino gli effetti che la narrazione può avere sul paziente e il proprio percorso di cura e di malattia. La narrazione ha un elevato potere evocativo e permette la rielaborazione del proprio vissuto mettendo in atto meccanismi riparativi e benefici (14), utili per lo stress derivato da eventi traumatici, come le situazioni di sofferenza e di malattia. Sarebbe interessante mettere a punto degli strumenti e dei metodi di ricerca che possano evidenziare questi aspetti, affinché il metodo narrativo trovi implementazione non solo come strumento di ricerca ma anche per il suo valore terapeutico.

\section{Disclosures}

Financial support: No financial support was received for this submission. Conflict of interest: The authors have no conflict of interest.

\section{Bibliografia}

1. CNMR. Relazioni degli esperti di consenso. http://www. iss.it/binary/cnmr/cont/Relazioni_degli_esperti_All. ConferenzaDiConsenso_MedicinaNarrativa.pdf (Accessed in date $3 / 9 / 2017)$.

2. Molzahn A, Sheilds L, Bruce A, Stajduhar K, Makaroff KS, Beuthin R, Shermak S. People living with serious illness: stories of spirituality. J Clin Nurs. 2012;21(15-16):2347-56.

3. Paterson JG, Zderad LT. Humanistic nursing. New York: John Wiley and Sons, 1976:42 e 44.

4. Vélez E, Ramasco M. Meaning of illness and illness representations, crucial factors to integral care. EDTNA ERCA J. 2006; 32(2):81-5.

5. Polaschek $\mathrm{N}$. The experience of living on dialysis: a literature review. Nephrol Nurs J. 2003;30(3):303-9, 313.

6. Kierans C. Narrating kidney disease: the significance of sensation and time in the emplotment of patient experience. Cul Med Psychiatry. 2005; 29(3): 341-59.

7. Curtin RB, Mapes D, Petillo M, Oberley E. Long-term dialysissurvivors: a transformational experience. Qual Health Res. 2002; 12(5):609-24.

8. Sheilds L, Molzahn A, Bruce A, Schick Makaroff K, Stajduhar K, Beuthin R, Shermak S. Contrasting stories of life-threatening illness: a narrative inquiry. Int J Nursing Stud. 2015; 52(1): 207-15

9. Kang $H$, Stenfors-Hayes T. Feeling Well and Having Good Numbers: Renal Patients' Encounter with Clinical Uncertainties and the Responsibility to "Live Well". Qual Health Res. 2016; 26(12):1591-602.

10. Kierans CM. Sensory and narrative identity: the narration of illness process among chronic renal sufferers in Ireland. NUI Maynooth - Anthropology \& Medicine. 2001;8(2-3):237-53.

11. Makaroff KL, Sheilds L, Molzahn A. Stories of chronic kidney disease: listening for the unsayable. J Adv Nurs. 2013; 69(12):2644-53.

12. Paley J (2009) Religion and secularization of health care. Journal of Clinical Nursing 18, 1963-1974.

13. Zola IK. Bringing our bodies and ourselves back in: Reflections on a past, present, and future medical sociology. Journal of Health and Social Behavior. 1991;32:1- 16.

14. Pennebaker JW, Seagal JD. Forming a story: the health benefits of narrative. J Clin Psychol. 1999;55:1243-5. 